\title{
Burkholderiapseudomallei: case series from the Provincial General Hospital, Kurunegala
}

WPH Abeydeera ${ }^{1}$, EM Corea $^{2}$, G Ranasinghe $^{1}$, G Nanayakkara $^{1}$, N Jayasekera ${ }^{1}, \mathrm{~N}_{\text {Munasinghe }}{ }^{1}$, JAAS.Jayaweera ${ }^{1}$

\section{Introduction}

Burkholderiapseudomallei, a Gram negative, bipolar-staining, oxidase-positive, non-fermenting bacterium is the aetiological agent of the clinical condition named melioidosis. It is a soil saprophyte, commonly causing infections in susceptible populations in agricultural areas. Infection is acquired by inoculation via breaches in the skin, inhalation of soil or ingestion of water contaminated by the bacterium. Melioidosis is common in tropical and subtropical regions of South East Asia and is an emerging pathogen. Sri Lanka also shares similar climatic conditions which result in vulnerability to the disease. The Kurunegala District in Sri Lanka is situated in the North Western Province which is an agricultural area with mainly paddy farming. Susceptible population includes farmers, diabetics and people with chronic kidney disease.

\section{Case series}

The Microbiology Laboratory of the Provincial General Hospital (PGH), Kurunegala isolated B. pseudomallei identified by latex agglutination and PCR at the Department of Microbiology, Faculty of Medicine, University of Colombo from 36 patients in a 15 month period from January 1st 2016. Of the 36 isolates, 21 were from blood culture in patients with septicaemia, 3 of whom died. Eleven isolates were from abscesses, one from a patient with septic arthritis and 3 from patients with bacteremia with no identified focus. Two patients had a positive culture from sputum. Seventeen patients of the 36 patients were had diabetes mellitus.

\section{Discussion and Conclusion}

Increased awareness among clinicians and improved diagnostic capability have contributed towards the increased diagnosis of melioidosis in Kurunegala Hospital. The majority of patients had a favourable clinical response. Active participation and collaboration with all stake holders will be continued and expanded so that patients with melioidosis presenting to this hospital would be facilitated by early diagnosis and initiation of appropriate treatment, thus reducing both morbidity and mortality associated with this disease.

\footnotetext{
${ }^{I}$ Provincial General Hospital, Kurunegala, Sri Lanka

${ }^{2}$ Faculty of Medicine, University of Colombo, Sri Lanka

Address for correspondence: Dr Harshula Abeydeera, Provincial General Hospital, Kurunegala, Sri Lanka. +94777268864 Email: harshulaabeydeera@yahoo.com 1 https://orcid.org/0000-0002-6156-7727
} 